\title{
NALTREXONE EFFECTS ON FOOD- AND CODEINE-MAINTAINED RESPONDING IN RHESUS MONKEYS
}

\section{SEYMORE HERLING *}

Departments of Pharmacology and Psychology, University of Michigan, Ann Arbor, Michigan 48109, U.S.A.

Received 3 April 1981, accepted 13 April 1981

S. HERLING, Naltrexone effects on food-and codeine-maintained responding in rhesus monkeys, European J. Pharmacol. 73 (1981) 41-49.

The delivery of food or the i.v. injection of codeine maintained lever pressing in rhesus monkeys during alternating periods of daily experimental sessions. Responding was maintained by food or codeine under a chain differential reinforcement of other behavior $30 \mathrm{sec}$ (DRO 30), fixed-ratio 30 response (FR 30) schedule of reinforcement. Maximum FR rates of codeine-reinforced responding were maintained by $25-47 \mu \mathrm{g} / \mathrm{kg}$ per injection codeine. Both lower $(8 \mu \mathrm{g} / \mathrm{kg}$ per injection) and higher $(80-800 \mu \mathrm{g} / \mathrm{kg}$ per injection) doses of codeine maintained lower FR response rates. FR rates of food-maintained responding only decreased as a function of codeine dose. Response rates during the DRO component of the schedule, when either food or codeine maintained responding, were extremely low $(<0.01$ responses/sec) and these rates were generally unaffected by the codeine dose. Naltrexone ( $0.003-0.32 \mathrm{mg} / \mathrm{kg}$ i.m.), administered before experimental sessions, produced a dose-related shift to the right in the rate-decreasing effects of codeine on FR responding maintained by food. In contrast, the doseeffect curve relating the FR rate of codeine-maintained responding to codeine dose was shifted consistently to the right only by the lowest dose of naltrexone $(0.003 \mathrm{mg} / \mathrm{kg})$. Although a 10 -fold higher dose of naltrexone, $0.03 \mathrm{mg} / \mathrm{kg}$, initially shifted the codeine self-injection curve to the right in one monkey, these higher naltrexone doses $(0.03-0.32 \mathrm{mg} / \mathrm{kg})$ generally resulted in response rates as low as or lower than those maintained by saline, across a wide range of codeine doses $(25-800 \mu \mathrm{g} / \mathrm{kg}$ per injection). These data suggest that naltrexone may decrease codeine-reinforced responding by mechanisms other than, or in addition to, a competitive antagonism of codeine's reinforcing effects.

Codeine Self-administration Naltrexone Rhesus monkeys

\section{Introduction}

The interactions between narcotic antagonists (e.g., naloxone, naltrexone) and narcotic self-administration depend on both past and current behavioral and pharmacological variables (e.g., Woods et al., 1973, 1975; Downs and Woods, 1975; Harrigan and Downs, 1978; Herling, 1981). For example, relatively large doses of naloxone $(1.0-3.2 \mathrm{mg} / \mathrm{kg})$ or naltrexone $(3.2 \mathrm{mg} / \mathrm{kg})$ decrease responding maintained by i.v. codeine to a greater extent

\footnotetext{
* Present address: National Institute on Drug Abuse, Addiction Research Center, P.O. Box 12390, Lexington, KY 40583, U.S.A.
}

than substituting saline for codeine (Woods and Schuster, 1971; Carney, 1976; Herling, 1981). Since one might predict that the complete antagonism of codeine by naloxone or naltrexone would produce an effect that mimicked that of saline substitution, narcotic antagonist-induced suppression of codeinereinforced responding may involve factors other than, or in addition to, antagonism of the reinforcing effects of codeine.

Analysis of the effects of narcotic antagonists on operant responding can be difficult since, with repeated injections of these drugs, a sensitivity to their effects can develop. For example, Kelleher and Goldberg (1979) showed that the daily injection of $10 \mathrm{mg} / \mathrm{kg}$ 
naloxone in monkeys shifted the dose-effect curve for the effects of naloxone on foodmaintained responding $\mathbf{1 0}$ fold to the left. This increased sensitivity to the effects of naloxone can last for periods of up to 4 years (Kelleher and Goldberg, 1979). A similar sensitization has been observed to the behavioral effects of naltrexone in rhesus monkeys (Herling, 1981). Thus, long-term studies involving repeated injections of narcotic antagonists may be complicated by changes in sensitivity to the effects of the antagonists.

The purpose of the present experiment was to examine the effects of relatively small doses of naltrexone on codeine-maintained responding across a wide range of codeine self-injection doses. Small doses of naltrexone were used to preclude the induction of sensitivity that can occur with the administration of high doses of narcotic antagonists. Lever pressing was maintained in rhesus monkeys during daily sessions under a multiple schedule of food and codeine reinforcement. Various i.v. doses of codeine, or saline, were made available during single sessions prior to which an i.m. injection of either saline or naltrexone was administered. Changes in behavior produced by changing the codeine dose that maintained responding were compared to changes induced by naltrexone pretreatment. Furthermore, the specificity of the pretreatment effects with respect to the reinforcer that maintained responding was assessed by evaluating, in the same subjects, the effects of naltrexone on food-reinforced responding. Food-reinforced responding also provided a baseline to assess the interaction between naltrexone and self-administered codeine on responding not maintained by a narcotic.

\section{Materials and methods}

\subsection{Subjects}

The subjects were two rhesus monkeys (Macaca mulatta) weighing 5.8 (monkey 1155) and $9.0 \mathrm{~kg}$ (monkey 808 ) prior to the start of the experiment. Monkey 808 had a history of food-maintained lever pressing and had received injections of various drugs for a period of approximately two years. This monkey, however, had neither participated in an experiment nor received any drugs for at least eight months prior to the start of this study. Monkey 1155 was experimentally and drug naive at the start of this study. Each monkey was reduced in weight to approximately $85 \%$ of its unrestricted-feeding weight and fed sufficient Purina Monkey Chow after each session to maintain its reduced weight. Fresh fruit was provided periodically. Each monkey had unrestricted access to water in its home cage and received a daily oral dose of $40 \mathrm{mg}$ isoniazid in a sugar cube.

Both monkeys were surgically prepared with indwelling siliconized rubber catheters (Rodhelm Reiss Co., Belle Mead, NJ), implanted in a jugular, femoral, or brachial vein; veins were used successively as needed. The distal end of the catheter passed under the skin and exited from the back of the animal in the midscapular area. Catheterizations were performed under ketamine $(10 \mathrm{mg} / \mathrm{kg}$ i.m.) and pentobarbital $(15 \mathrm{mg} / \mathrm{kg}$ i.v.) anesthesia. Leather vests were worn to protect the catheter.

\subsection{Apparatus}

The apparatus used in this study has been described in detail previously (Herling, 1981). Before each daily session, each monkey was removed from its individual home cage and placed in a restraint chair. Monkey and chair were then placed in a sound-attenuating wooden chamber, fifteen min prior to the start of the session. A metal console, located in the center of the front wall of the chamber, contained a response lever (BRS/LVE, Beltsville, MD, model PRL-001/121-07) and a food cup into which $300 \mathrm{mg}$ pellets (P.J. Noyes Co., Lancaster, NH, Formula G, banana flavored) could be delivered by a food pellet dispenser (Ralph Gerbrands Co., Arlington, 
MA, model A) that was located outside the chamber. Various colored stimulus lights ( $7-\mathrm{W}$, General Electric Co., model C7-CC), mounted at the top of the front wall of the chamber, were correlated with different components of the session. A motor-driven roller pump (Watson-Marlow Ltd., Falmouth, England, model MHRK 55) delivered drug solutions to the monkey from a reservoir through siliconized rubber tubing and the indwelling catheter. Both the pump and reservoir were located outside the experimental chamber. Scheduling of events and collection of data were accomplished with a Texas Instruments $960 \mathrm{~A}$ digital computer and cumulative recorders (Ralph Gerbrands Co., Arlington, MA, model C).

\subsection{Procedure}

Each monkey was trained to press a lever thirty times (fixed-ratio 30, FR 30) in the presence of two blue lights in order to obtain a single $300 \mathrm{mg}$ banana-flavored food pellet. A $5 \mathrm{sec}$ time-out period (TO $5 \mathrm{sec}$ ), during which no lights were on in the chamber, followed each food presentation. Responding during the TO period had no scheduled consequences. A differential reinforcement of other behavior (DRO) component, signalled by a pair of yellow lights, followed the TO. During the DRO component, the animal was required not to press the lever for $30 \mathrm{sec}$ (DRO $30 \mathrm{sec}$ ) in order to advance the schedule into the FR component. Food pellets were delivered only after completion of the $F R$ requirement. Responses during the DRO component delayed the onset of the FR component by 30 sec. In addition, the animals were required to complete each FR 30 within $60 \mathrm{sec}$ of the onset of the blue stimulus lights (limited-hold $60 \mathrm{sec}, \mathrm{LH} \mathrm{60)}$ ). If 30 responses were not completed in time, food was not delivered and the schedule advanced to the next DRO component. The schedule of reinforcement can be designated as a chain DRO $30 \mathrm{sec}, \mathrm{FR} 30$ schedule of food presentation.

Initially, each session consisted of three periods of food availability, separated by two
$22.5 \mathrm{~min}$ TO periods. During these TO periods, the chamber was unlighted and responding had no scheduled consequences. Each food-availability period began with the FR component of the schedule and ended after the fifteenth DRO $30 \mathrm{sec}$ component.

When responding under the schedule of food presentation was stable, 5 -sec intravenous injections of $80 \mu \mathrm{g} / \mathrm{kg}$ per injection of codeine were made available under a chain DRO 30 sec (red light) FR 30 (green light) schedule of reinforcement during the two $22.5 \mathrm{~min}$ TO periods that had previously separated periods of food availability. The $60 \mathrm{sec}$ limited hold was also in effect during these codeine-availability periods. The i.v. delivery of codeine occurred when the chamber was unlighted during a $5 \mathrm{sec}$ period following each completed FR 30, which corresponded to the $5 \mathrm{sec}$ TO following each delivery of a food pellet during the food-availability periods. Each codeine-availability period began with the FR component of the schedule and ended after the fifteenth DRO $30 \mathrm{sec}$ component. If a codeine-availability period ended before $22.5 \mathrm{~min}$ elapsed, the animal spent the remaining time before the next food-availability period in a TO. Thus, an entire session consisted of three periods of food availability, separated by two periods of codeine availability. A variable period of TO $(0-13.75 \mathrm{~min}$, depending on the monkey's performance), separated a codeine-availability and the next food-availability period of the session.

When performances maintained by food and codeine $(80 \mu \mathrm{g} / \mathrm{kg}$ per injection) were stable, the effects of substituting one of a range of doses of codeine $(8-800 \mu \mathrm{g} / \mathrm{kg}$ per injection) or saline, and the effects of presession i.m. injections of naltrexone (0.003$0.32 \mathrm{mg} / \mathrm{kg}$ ) or saline were determined during single sessions. At least one session, during which food and $80 \mu \mathrm{g} / \mathrm{kg}$ per injection codeine maintained responding and no presession injection was administered intervened between dose substitution and naltrexone pretreatment sessions. 


\subsection{Drugs}

All drugs were dissolved in sterile saline $(0.9 \% \mathrm{NaCl})$. Naltrexone hydrochloride (Endo Laboratories, Garden City, NY) was injected i.m. in a volume of $0.2 \mathrm{ml} / \mathrm{kg}$ body weight immediately prior to placing the monkey in the experimental chamber; i.e., fifteen min before the start of the session. Each $5 \mathrm{sec}$ i.v. injection of codeine phosphate (S.B. Penick and Co., Lyndhurst, NJ) or codeine hydrochloride (Dr. R.E. Willette, NIDA) was delivered in a volume of $1 \mathrm{ml}$. The dose of self-injected codeine for each animal was adjusted by altering the concentration of the codeine solution. Codeine doses are expressed in terms of the base; doses of naltrexone refer to the salt form.

\section{Results}

When food alone maintained responding, response rates during the $\mathrm{FR}$ component of the schedule in both monkeys ranged from 3.0 to 4.0 responses/sec. Response rates during the TO and DRO components were considerably lower, averaging 0.4 and 0.01 responses/sec, respectively. The pattern of responding generated by the schedule was similar to that previously described under this schedule of reinforcement (Woods et al., 1978; Herling, 1981). Briefly, in the presence of the FR stimulus lights, responding occurred at a high steady rate until food was delivered. This was usually followed by a pause in responding during the TO and DRO components of the schedule, although a few responses occasionally occurred during these components of the schedule. Immediately following the DRO component of the schedule, the stimulus lights correlated with the FR component were illuminated and responding again occurred at a high steady rate until food delivery.

Codeine-reinforced responding was established in both monkeys in alternate components of the session with the i.v. injection of

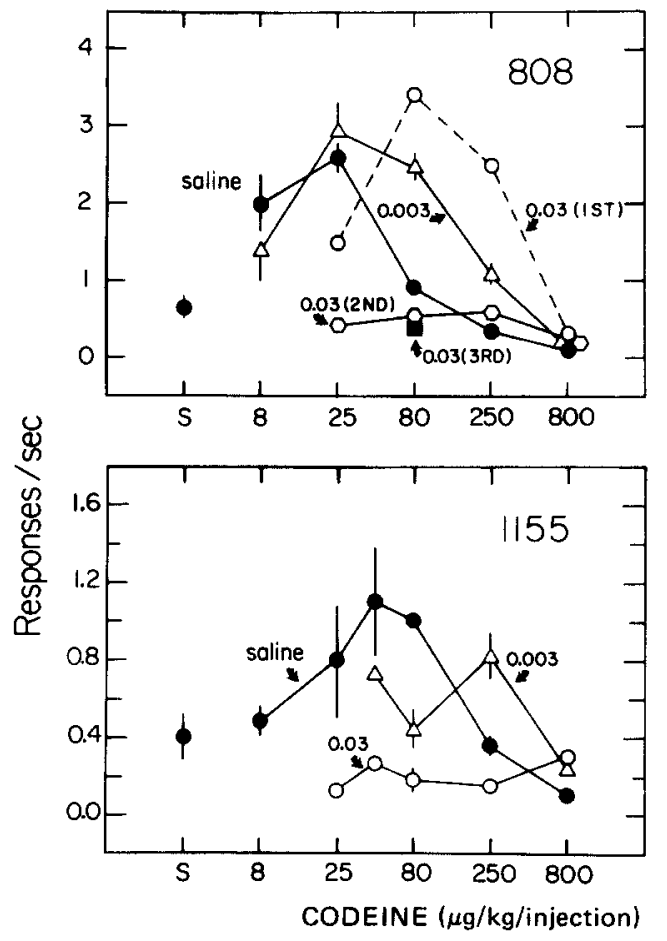

Fig. 1. Fixed-ratio rates of responding maintained by codeine as a function of dose per injection during sessions prior to which i.m. saline or naltrexone was administered. Ordinates: response rate, responses/sec. Abscissae: i.v. codeine dose, $\mu \mathrm{g} / \mathrm{kg}$ per injection. For monkey 808 , points representing the effects of $0.03 \mathrm{mg} / \mathrm{kg}$ naltrexone indicate data from single sessions. First (1st), second (2nd) and third (3rd) determinations of the effects of $0.03 \mathrm{mg} / \mathrm{kg}$ naltrexone at different codeine doses are shown separately for this monkey. All other points are the mean of four (saline) or two (naltrexone) observations. Refer to table 1 for exceptions. Lines through the points indicate \pm 1 S.E.M. Points at $\mathbf{S}$ represent rates of responding maintained by saline. Note difference in the scale of the ordinates for the two monkeys.

$80 \mu \mathrm{g} / \mathrm{kg}$ codeine. The pattern of responding maintained by codeine was similar to that maintained by food. Relatively high rates of responding occurred during the FR component of the schedule. In contrast, few responses occurred during either the TO $(<0.4$ responses/ $\mathrm{sec})$ or DRO $(<0.01$ responses/sec) components of the schedule.

Codeine, $80 \mu \mathrm{g} / \mathrm{kg}$ per injection, maintained average FR response rates of approxi- 


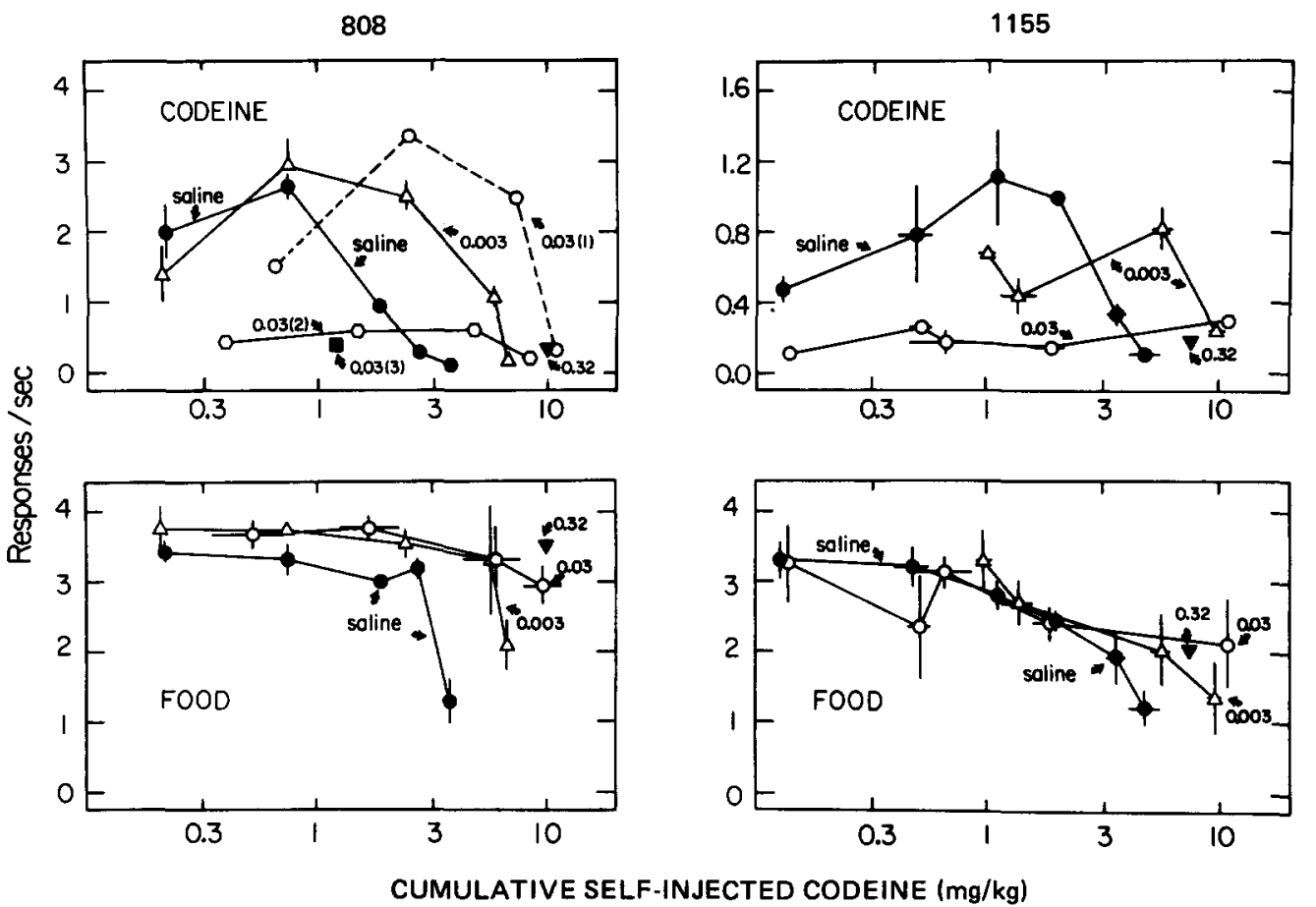

Fig. 2. Fixed-ratio rates of responding maintained by codeine (upper panels) and food (lower panels) as a function of the cumulative self-injected i.v. dose of codeine during sessions prior to which i.m. saline or naltrexone was injected. Ordinates: response rate, responses/sec. Abscissae: cumulative i.v. codeine dose per session, $\mathrm{mg} / \mathrm{kg}$. For monkey 808, the points indicating the first (1), second (2) and third (3) determinations of the effects of $0.03 \mathrm{mg} /$ $\mathrm{kg}$ naltrexone on codeine-maintained responding (upper panel) are data from single sessions. Otherwise each point is the mean of four (saline) or two (naltrexone) observations. Refer to table 1 for exceptions. Vertical lines through the points indicate \pm 1 S.E.M. of the response rate. Horizontal lines through the points indicate \pm 1 S.E.M. of the cumulative codeine dose. Note difference in the scale of the ordinate for codeine-maintained responding in monkey 1155.

mately 1.0 response/sec in both monkeys (fig. 1). When higher doses of codeine (250$800 \mu \mathrm{g} / \mathrm{kg}$ per injection) were substituted, rates of responding maintained by codeine decreased. On the other hand, as the dose of codeine that maintained responding was reduced below $80 \mu \mathrm{g} / \mathrm{kg}$ per injection, FR response rates first increased to a maximum maintained by 25 and $47 \mu \mathrm{g} / \mathrm{kg}$ per injection in monkeys 808 and 1155 , respectively, and then decreased (fig. 1 , closed circles). When saline was substituted for codeine, FR response rates maintained by saline occurred at high rates early in the session, but as the session progressed, rates of responding decreased to near zero responses/sec. Average saline-maintained FR response rates for the entire session were approximately 0.7 (monkey 808) and 0.4 (monkey 1155) responses/sec (fig. 1, symbols above $\mathrm{S}$ ). FR responding maintained by food during these sessions occurred at high rates throughout the session, averaging approximately 3.5 responses/sec.

Response rates maintained by food or codeine are shown as a function of the cumulative self-injected dose of codeine in fig. 2 (closed circles). As the cumulative selfinjection dose increased, codeine-maintained FR response rates increased to a maximum, 
TABLE 1

Codeine intake $(\mathrm{mg} / \mathrm{kg})$ per session following presession injections of saline or naltrexone ${ }^{\mathrm{a}}$.

\begin{tabular}{|c|c|c|c|c|c|}
\hline \multirow[t]{3}{*}{ Monkey No. } & \multirow{3}{*}{$\begin{array}{l}\text { Codeine } \\
\mu \mathrm{g} / \mathrm{kg} \text { per } \\
\text { injection }\end{array}$} & \multicolumn{4}{|c|}{ Presession injection } \\
\hline & & \multirow[t]{2}{*}{ Saline } & \multicolumn{3}{|c|}{ Naltrexone, $\mathrm{mg} / \mathrm{kg}$} \\
\hline & & & 0.003 & 0.03 & 0.32 \\
\hline \multirow[t]{5}{*}{808} & 8 & $0.22 \pm 0.01$ & $0.21 \pm 0.02$ & - & - \\
\hline & 25 & $0.75 \pm 0.01$ & $0.75 \pm 0.00$ & $0.53 \pm 0.18$ & - \\
\hline & 80 & $1.86 \pm 0.02^{6}$ & $2.44 \pm 0.06^{3}$ & $1.74 \pm 0.49^{3}$ & - \\
\hline & 250 & $2.75 \pm 0.18$ & $5.75 \pm 0.00$ & $6.00 \pm 1.70$ & - \\
\hline & 800 & $3.78 \pm 0.28$ & $6.72 \pm 0.00$ & $9.66 \pm 1.80$ & $10.08^{1}$ \\
\hline \multirow[t]{6}{*}{1155} & 8 & $0.13 \pm 0.01$ & - & - & - \\
\hline & 25 & $0.49 \pm 0.08$ & - & $0.14 \pm 0.01$ & - \\
\hline & 47 & $1.13 \pm 0.09$ & $0.99 \pm 0.06$ & $0.52 \pm 0.06$ & - \\
\hline & 80 & $2.01 \pm 0.03^{6}$ & $1.37 \pm 0.24^{3}$ & $0.67 \pm 0.21^{3}$ & - \\
\hline & 250 & $3.63 \pm 0.28$ & $5.69 \pm 0.49^{4}$ & $1.88 \pm 0.344$ & - \\
\hline & 800 & $4.83 \pm 0.73$ & $9.66 \pm 0.63^{4}$ & $11.13 \pm 0.73^{4}$ & $7.56^{1}$ \\
\hline
\end{tabular}

a Each value represents the mean \pm 1 S.E.M. for four (saline) or two (naltrexone) observations, unless otherwise indicated by superscript numerals.

and then decreased (fig. 2, upper panels). In contrast, FR rates of responding maintained by food only decreased as a function of the cumulative self-injected codeine dose (fig. 2 , lower panels). The low rates of responding during the TO and DRO components, whether food or codeine maintained responding, were generally unaffected by the codeine dose (not shown).

In both monkeys, codeine intake per session increased as the codeine dose per injection increased, whether saline or various doses of naltrexone were injected prior to the session (table 1). With all naltrexone pretreatment doses, codeine intake was unchanged or decreased at the lower codeine doses and increased at the higher codeine doses. At the highest injection dose of codeine $(800 \mu \mathrm{g} / \mathrm{kg}$ per injection), naltrexone $(0.003-0.32 \mathrm{mg} /$ $\mathrm{kg}$ ) increased codeine intake $57-166 \%$.

The effects of presession i.m. injections of naltrexone $(0.003-0.32 \mathrm{mg} / \mathrm{kg})$ on the average $F R$ rate of responding maintained by codeine are shown in fig. 1 as a function of the dose/injection of codeine and in fig. 2 (upper panels) as a function of cumulative self-injection dose. The lowest dose of naltrexone $(0.003 \mathrm{mg} / \mathrm{kg})$ had little effect on FR rates of responding maintained by the lower injection doses of codeine in monkey 808 , but decreased the maximum rates of responding maintained by intermediate doses of codeine $(47-80 \mu \mathrm{g} / \mathrm{kg}$ per injection) in monkey 1155 (fig. 1). In both monkeys, $0.003 \mathrm{mg} / \mathrm{kg}$ naltrexone increased the lower response rates maintained by certain higher injection doses of codeine; i.e., the doseeffect curve relating $F R$ response rate to codeine injection dose was shifted to the right by $0.003 \mathrm{mg} / \mathrm{kg}$ naltrexone.

In monkey 808 , presession injection of a 10 -fold higher dose of naltrexone, $0.03 \mathrm{mg} /$ $\mathrm{kg}$, also shifted the codeine self-injection curve to the right. This effect with $0.03 \mathrm{mg} /$ $\mathrm{kg}$ naltrexone, however, was observed only on the first determination of this dose-effect curve in this monkey. On subsequent administrations of $0.03 \mathrm{mg} / \mathrm{kg}$ naltrexone in monkey 808 , and on each determination in monkey $1155,0.03 \mathrm{mg} / \mathrm{kg}$ naltrexone only decreased responding maintained by low to intermediate doses of codeine and had little effect on low response rates maintained by higher codeine doses. Similar dose-related effects of 
naltrexone were obtained when FR response rates were plotted as a function of cumulative codeine dose (fig. 2, upper panels). Thus, following a pretreatment with $0.03 \mathrm{mg} / \mathrm{kg}$ naltrexone, rates of responding maintained by a wide range of codeine doses $(25-800 \mu \mathrm{g} / \mathrm{kg}$ per injection) were as low as or lower than response rates maintained by saline (fig. 1). During these sessions, responding maintained by food occurred at a high rate throughout the session (>2 responses/sec), whereas responding maintained by codeine occurred at a high rate initially, but as the session progressed, response rates decreased to near zero responses/sec. Thus, the effects of pretreatment with naltrexone $(0.03 \mathrm{mg} / \mathrm{kg})$ on the rate and pattern of responding resembled, in all important respects, the effects of substituting saline for codeine in the session. Increasing the naltrexone pretreatment dose to $0.32 \mathrm{mg} / \mathrm{kg}$ had little effect on the low FR response rates maintained by $800 \mu \mathrm{g} / \mathrm{kg}$ per injection of codeine (fig. 2, upper panels; closed triangles).

Although naltrexone $(0.03-0.32 \mathrm{mg} / \mathrm{kg})$ did not increase the low $F R$ response rates maintained by high doses of codeine (fig. 1; fig. 2, upper panels), low rates of food-maintained responding that occurred when the codeine dose was high, were increased by naltrexone (fig. 2, lower panels). For example, when the cumulative codeine dose was greater than $3 \mathrm{mg} / \mathrm{kg}$, response rates maintained by food were approximately 1.1 responses/sec in both monkeys. On the other hand, in the presence of naltrexone $(0.003-0.32 \mathrm{mg} / \mathrm{kg})$, foodmaintained response rates were between 2 and 3 responses/sec, even when cumulative doses of up to $10 \mathrm{mg} / \mathrm{kg}$ codeine were self-administered.

\section{Discussion}

In the absence of naltrexone pretreatment, as the dose of codeine increased, response rates maintained by codeine increased to a maximum and then decreased. This biphasic relationship between response rate and dose of self-administered drug is common in narcotic self-administration studies when sufficiently wide dose ranges are examined (e.g., Woods and Schuster, 1968; Hoffmeister and Schlichting, 1972; Downs and Woods, 1974; Harrigan and Downs, 1978; Young and Woods, 1980; Herling, 1981). Rates of foodreinforced responding, on the other hand, only decreased as a function of the cumulative codeine dose.

The lowest dose of naltrexone $(0.003 \mathrm{mg} /$ $\mathrm{kg}$ ) shifted the descending limb of the codeine self-injection curve to the right in both monkeys and decreased maximum response rates maintained by codeine in monkey 1155 . In the other subject, monkey 808 , a higher dose of naltrexone $(0.03 \mathrm{mg} / \mathrm{kg}$; first determination) decreased the maximum response rates maintained by $25 \mu \mathrm{g} / \mathrm{kg}$ per injection codeine and increased the lower rates maintained by higher codeine doses. Thus, in both monkeys, the dose of codeine that maintained maximum rates of responding could be shifted to the right by an appropriate dose of naltrexone $(0.003 \mathrm{mg} / \mathrm{kg}$ : monkey 1155 ; $0.03 \mathrm{mg} / \mathrm{kg}$, first determination: monkey 808 ).

On the other hand, higher doses of naltrexone $(0.03-0.32 \mathrm{mg} / \mathrm{kg})$ generally decreased responding maintained by low to intermediate doses of codeine and had little effect on the rate of responding maintained by higher codeine doses. Thus, in both monkeys, no dose of codeine, in the presence of these naltrexone doses, maintained response rates above those maintained by saline. These naltrexone-induced changes in codeine-reinforced responding occured at doses of the antagonist $(0.003-0.32 \mathrm{mg} / \mathrm{kg})$ that are $30-3000$ times lower than those needed to affect food-reinforced responding (e.g., Herling, 1981). Moreover, by restricting the magnitude of the naltrexone pretreatment doses, the increased sensitivity to the ratedecreasing effects of narcotic antagonists on food-reinforced responding that occurs with repeated exposures to relatively high doses of these drugs (e.g., $10 \mathrm{mg} / \mathrm{kg}$ naloxone: 
Kelleher and Goldberg, 1979; $3.2 \mathrm{mg} / \mathrm{kg}$ naltrexone: Herling, 1981) was not observed in the present experiment. For example, a dose of naltrexone as low as $0.32 \mathrm{mg} / \mathrm{kg}$ is capable of completely suppressing both foodand codeine-maintained responding following repeated injections of $3.2 \mathrm{mg} / \mathrm{kg}$ naltrexone (Herling, 1981). In the present study, however, by judiciously selecting the naltrexone pretreatment doses, $0.32 \mathrm{mg} / \mathrm{kg}$ naltrexone increased codeine intake 1.5-2.5 times and only reversed the rate-decreasing effects of codeine on food-maintained responding.

Since naltrexone, $0.03-0.32 \mathrm{mg} / \mathrm{kg}$, produced effects on codeine-maintained responding that resembled in all important aspects the effects of substituting saline for codeine, these changes in codeine-maintained responding can be interpreted as an antagonism by naltrexone of the reinforcing effects of codeine. One might have predicted, however, when higher doses of codeine maintained low rates of responding, that appropriate doses of naltrexone would have produced effects similar to those produced by substituting certain lower doses of codeine, i.e., that naltrexone would have produced increases in the low rates of responding maintained by high codeine doses. In the present experiment, a 100-fold range of naltrexone doses $(0.003-0.32 \mathrm{mg} / \mathrm{kg})$ had little effect on the rate of responding maintained by $800 \mu \mathrm{g} / \mathrm{kg}$ per injection codeine, although total codeine intake was increased.

These results confirm previous observations (Harrigan and Downs, 1978; Herling, 1981) that naltrexone can suppress narcotic-reinforced responding to levels at or below those maintained by saline, across a wide range of narcotic self-injection doses. For example, Harrigan and Downs (1978) showed that increasing the continuously infused i.v. dose of naltrexone $(0.024-0.48 \mathrm{mg} / \mathrm{kg}$ per day) increased the dose of morphine that maintained the maximum injection rate. However, as the naltrexone dose was increased, the maximum number of injections of morphine taken by the subjects decreased. At the highest naltrexone infusion rates $(0.12-$ $0.48 \mathrm{mg} / \mathrm{kg}$ per day) no dose of morphine was injected at a rate above that maintained by saline. Since high response rates maintained by intermediate doses of morphine were decreased by naltrexone relatively more than low response rates maintained by high morphine doses were increased, it was suggested that naltrexone antagonizes the reinforcing effects of morphine to a greater extent than it antagonizes the rate-decreasing effects of morphine (Harrigan and Downs, 1978). A similar argument might be made for the effects of naltrexone on codeine self-administration in the present experiment. However, doses of naltrexone that had little or no effect on the low rates of responding maintained by high doses of codeine, antagonized the ratedecreasing effects of these codeine doses when food maintained responding in the same animals (fig. 2). Thus, under the conditions of the present experiment, the ability of naltrexone to reverse the rate-decreasing effects of codeine appeared to depend on the response-maintaining event (i.e., food vs. codeine).

The inability of naltrexone to increase low rates of responding maintained by high codeine doses suggests, perhaps, that these doses of codeine, in the presence of presession injections of naltrexone, punish responding. A similar possibility was advanced by Harrigan and Downs (1978) to account for the inability of naltrexone to increase responding maintained by high doses of morphine. Along these lines, Woods and Schuster (1971) suggested that self-injected codeine may be aversive in the presence of the narcotic antagonist naloxone, since the effect of naloxone pretreatment was to suppress codeine-reinforced responding considerably more than simply substituting saline for codeine. Additionally, it has been shown that responding maintained by i.v. codeine is suppressed when responding results in the delivery of both codeine and naloxone. Recovery from such suppression requires several sessions, suggesting that punishment is involved when 
responding produces naloxone-codeine combinations (Woods et al., 1975). Perhaps naloxone blocks the reinforcing effects of codeine allowing a non-narcotic action of codeine to exert a punishing effect on responding.

In the present experiment, presession injections of relatively low doses of naltrexone resulted in response rates maintained by a wide range of codeine doses that were similar to or below those maintained by saline, suggestive of antagonism by naltrexone of the reinforcing effects of codeine. Additionally, naltrexone, at doses that increased food-maintained responding that had been decreased by codeine, failed to increase low response rates maintained by high doses of codeine. As noted above, naltrexone-induced increases in responding maintained by high doses of codeine might have been expected if the effects of naltrexone pretreatment were similar to the effects of substituting appropriate lower doses of codeine. The suppression of codeine-reinforced responding by naltrexone, particularly when high doses of codeine maintain responding, may involve punishment in addition to an antagonism of codeine's reinforcing effects.

\section{Acknowledgements}

This research was supported by USPHS Grant DA 00154 (James H. Woods, Principal Investigator) and is based on a dissertation submitted by the author to the Graduate School of the University of Michigan in partial fulfillment of the requirements for a Ph.D. degree in the Department of Psychology. The author wishes to express his gratitude to James $\mathrm{H}$. Woods and Gail Winger for their guidance and support throughout the course of this study and to Jonathan L. Katz, Robert E. Solomon and Alice M. Young for comments and suggestions on the manuscript. The assistance of Isabel Herling and Elaine Sudman in preparing the manuscript is gratefully acknowledged.

\section{References}

Carney, J.M., 1976, Selective Modulation of CodeineReinforced Responding in Rhesus Monkeys, Doctoral Dissertation, University of Michigan, (Univer- sity Microfilms International, Ann Arbor, Michigan).

Downs, D.A. and J.H. Wōods, 1974, Codeine- and cocaine-reinforced responding in rhesus monkeys: Effects of dose on response rates under a fixedratio schedule, J. Pharmacol. Exp. Ther. 191, 179.

Downs, D.A. and J.H. Woods, 1975, Naloxone as a negative reinforcer in rhesus monkeys: Effects of dose, schedule, and narcotic regimen, Pharmacol. Rev. 27, 397.

Harrigan, S.E. and D.A. Downs, 1978, Continuous intravenous naltrexone effects on morphine selfadministration in rhesus monkeys, J. Pharmacol. Exp. Ther. 204, 481.

Herling, S., 1981, Effects of naltrexone dose and history of naltrexone exposure on food- and codeine-maintained responding in rhesus monkeys. J. Pharmacol. Exp. Ther. 217, 105.

Hoffmeister, F. and U.U. Schlichting, 1972, Reinforcing properties of some opiates and opioids in rhesus monkeys with histories of cocaine and codeine self-administration, Psychopharmacol. 23, 55.

Kelleher, R.T. and S.R. Goldberg, 1979, Effects of naloxone on schedule-controlled behavior in monkeys, in: Endorphins in Mental Health Research, eds. E. Usdin, W.E. Bunney, Jr. and N.S. Kline (Oxford University Press, New York) p. 461.

Woods, J.H., D.A. Downs and J. Carney, 1975, Behavioral functions of narcotic antagonists: response-drug contingencies, Federation Proc. 34, 1777.

Woods, J.H., D.A. Downs and J.E. Villarreal, 1973, Changes in operant behavior during deprivationand antagonist-induced withdrawal states, in: Bayer-Symposium IV, Psychic Dependence: Definition, Assessment in Animals and Man; Theoretical and Clinical Implications, eds. L. Goldberg and F. Hoffmeister (Springer-Verlag, Berlin) p. 114.

Woods, J.H., S. Herling and G. Winger, 1978, Chlorpromazine- and haloperidol-induced changes in some pehavioral effects of cocaine and amphetamine, in: Neuro-psychopharmacology: Proceedings of the Tenth Congress of the Collegium Internationale Neuro-psychopharmacologicum, V. 2, eds. P. Deniker, C. Radouco-Thomas and A. Villeneuve (Pergamon, Oxford) p. 1485.

Woods, J.H. and Schuster, C.R., 1968, Reinforcement properties of morphine, cocaine and SPA as a function of unit dose, Int. J. Addict. 3, 231.

Woods, J.H. and Schuster, C.R., 1971, Opiates as reinforcing stimuli, in: Stimulus Properties of Drugs, eds. T. Thompson and R. Pickens (AppletonCentury-Crofts, New York) p. 163.

Young, A.M. and Woods, J.H., 1980, Behavior maintained by intravenous injection of codeine, cocaine, and etorphine and the rhesus macaque and the pigtail macaque, Psychopharmacol. 70, 263. 